\title{
Testing times for gene test regulators
}

\author{
The Obama administration's hint at regulatory rollback may make stricter oversight of direct-to-consumer (DTC) \\ genetic testing less inevitable, particularly as fresh evidence on consumer attitudes suggests buyers can handle \\ the information.
}

ast summer, the US Food and Drug Administration (FDA) sent Lletters to several companies warning them that their genetic testing products were devices that were not FDA-approved. This shot across the bow, together with a withering Congressional hearing and critical Government Accountability Office report, aimed as it was at firms such as 23andMe, Consumer Genetics, CyGene Direct, deCODEme, Navigenics, Pathway Genomics and Sequenom, which offer tests consumers can buy directly, seemed to signal the beginning of the end of the DTC business model. All may not be lost, however. Two seemingly unconnected developments in recent weeks-one political and the other in social science-may make prediction of the field's demise premature.

On January 18, US president Barack Obama issued an executive order requesting all US government arms, including the FDA, to review regulations pertaining to business. The goal is to change, streamline, expand or repeal anachronistic rules that might smother US efforts to restore its economy, jobs and international competitiveness. Much to the delight of some Obama opponents-and much to the discomfort of public citizen NGOs-2011 seems to have ushered in a lighter regulatory touch for the administration: regulate, but only rationally and when necessary.

In an accompanying op-ed piece in the Wall Street Journal, President Obama also asked US agencies to have "increased flexibility" when it comes to reducing regulatory burdens on small businesses. $\mathrm{He}$ invoked the 1980 Regulatory Flexibility Act that formalized the ways the administration can help out small businesses. These include simplified reporting, the use of performance standards rather than design standards, streamlining of filing and even exemption from certain rules.

For DTC genetics companies this could be a potentially significant fillip. Particularly in light of a study published in the January 12 issue of the New England Journal of Medicine by researchers from Scripps Translational Science Institute. This study involved over 2,000 people drawn from high-technology companies in the San Diego area who had paid (in fact, co-paid with their companies' health plans) to use the Navigenic Health Compass single nucleotide polymorphism (SNP) profiling system. As the subjects signed up for profiling, their anxiety levels were assessed before testing and after they had received the results; any major lifestyle changes made or medical interventions sought after testing were also noted.

Until this study, most of the previous work on the psychological impact of genetic testing has lacked sufficient intellectual rigor and/ or numerical powering to provide conclusive insights. The Scripps study involved at least ten times more subjects than previous work, used standard psychological research instruments, was conducted with the involvement of psychology professionals and at arms-length from the genetic profiling companies.

What the authors found was that genome-wide SNP profiles have no statistically significant impact on the anxiety levels of subjects. Indeed, the average anxiety score diminished slightly after the results were known from what was already a low-anxiety reading before testing. Over $90 \%$ of individuals had no anxiety over the results.

The Navigenics Health Compass readout is, in essence, a simple color-coded dashboard matrix showing a subject's lifetime risk of some 20 common conditions, such as various types of cancers, multiple sclerosis, lupus or diabetes. The patient's own profiled risk is compared with that of the general population and any susceptibilities significantly above population risk are coded with an orange warning tint.

Rather than being frightened by any flagged susceptibilities, a significant percentage of those tested took some form of low-level action as a result of receiving their results. A few people cut down on cigarette or alcohol consumption (although others increased their intakes). Some exercised more or changed their diet, whereas others promised themselves that they would. And over a quarter of the subjects consulted with their primary care physicians, profile readouts in hand.

And this is where the problem lay. According to both the Scripps authors and Navigenics, $90 \%$ of physicians remain uncomfortable with dealing with profiling data. It is not that they have any huge conceptual misgivings about the approach or, indeed, that they are nervous about dealing with 'empowered' patients. It's simply that they do not know the extent to which any of the test data has been medically validated.

There are some caveats. First and foremost, the subjects recruited for the study work at high-tech companies and are typical of early adopters but not representative of the public at large. Second, the findings are subject to the biases of web-based, self-reported assessment. And third, the study was based on a single, short-term assessment, rather than long-term follow-up.

Nevertheless, the Scripps data provide at least one pointer to the Obama Administration and the FDA that their stance to the DTC genetics sector has been perhaps too aggressive. It is not the genomewide SNP scans developed by companies for consumers that need regulation, it is the genetic tests used by physicians for medical decision-making that require more stringent clinical validation and closer regulatory scrutiny.

This journal has long contended that the best way forward for gene test regulation would be to refine existing Clinical Laboratory Improvement Amendments (CLIA) rules for laboratory-developed genetic tests and FDA $510(\mathrm{k})$ oversight of in vitro diagnostics and devices so that medical practitioners can have greater confidence in the analytical validity of a genetic test (that is, does the test find what it's supposed to?).

At the same time, in the light of President Obama's call for regulatory precision for small businesses and present evidence from users, an FDA clampdown on DTC genetics companies appears unwarranted. Although early adopters are still, broadly speaking, uncertain about what they want from genetic profiling, it seems they are relatively unconcerned. A big unanswered question is will the broader public feel the same? 